\title{
Modeling Complex Systems by Structural Invariants Approach
}

\author{
Jiri Bila $[$, , Ali. H. Reshak $(\mathbb{D}$, and Jan Chysky $\mathbb{1}$ \\ Czech Technical University in Prague, Institute of Instrumentation and Control Engineering, Technicka 4, 16607 Prague 6, \\ Czech Republic
}

Correspondence should be addressed to Jiri Bila; jiri.bila@fs.cvut.cz

Received 11 December 2020; Revised 13 June 2021; Accepted 19 July 2021; Published 6 September 2021

Academic Editor: Chrystopher L. Nehaniv

Copyright (C) 2021 Jiri Bila et al. This is an open access article distributed under the Creative Commons Attribution License, which permits unrestricted use, distribution, and reproduction in any medium, provided the original work is properly cited.

\begin{abstract}
When modeling complex systems, we usually encounter the following difficulties: partiality, large amount of data, and uncertainty of conclusions. It can be said that none of the known approaches solves these difficulties perfectly, especially in cases where we expect emergences in the complex system. The most common is the physical approach, sometimes reinforced by statistical procedures. The physical approach to modeling leads to a complicated description of phenomena associated with a relatively simple geometry. If we assume emergences in the complex system, the physical approach is not appropriate at all. In this article, we apply the approach of structural invariants, which has the opposite properties: a simple description of phenomena associated with a more complicated geometry (in our case pregeometry). It does not require as much data and the calculations are simple. The price paid for the apparent simplicity is a qualitative interpretation of the results, which carries a special type of uncertainty. Attention is mainly focused (in this article) on the invariant matroid and bases of matroid $(M, \mathrm{BM})$ in combination with the Ramsey graph theory. In addition, this article introduces a calculus that describes the emergent phenomenon using two quantities-the power of the emergent phenomenon and the complexity of the structure that is associated with this phenomenon. The developed method is used in the paper for modeling and detecting emergent situations in cases of water floods, traffic jams, and phase transition in chemistry.
\end{abstract}

\section{Introduction}

The field of complex systems is proving to be a much needed field of research. In this article, we focus mainly on modeling complex systems and processing emergent situations.

Many approaches have been used for modeling complex systems until now and here we introduce some of them:

Multi-agent and cellular automata approach $[1,2]$

Physical approach [3]

Probability and statistical approach [4]

State approach $[5,6]$

Complexity and entropy approach [7-9]

Structural invariant approach [10]

Simulation approach $[11,12]$

Each of the introduced approaches has advantages and disadvantages and could be characterized by many pages.
In this article, we concentrate especially on emergent behavior and emergent situations in complex networks of transport and hydrological systems and on systems of material physics and chemistry.

This article has been inspired, among other sources, by the book, "A Different Universe (Reinventing Physics from the bottom down), by Robert B. Laughlin (Nobel Prize winner)" [13]. Laughlin introduces "emergence as an organization principle" and from this point of view explores a wide field of physics. The whole book leads to the following opinion: "If we accept the world with emergence, we also announce the end of reductionism. (That is - the end of the time when we thought that everything essential in the world could be calculated.)" This means that when studying such systems, there is always a certain part of the events that we cannot calculate.

In this article, we try to estimate (qualitatively) how big this part of the story is, which we cannot calculate. As will 
be discussed below, complex systems with emergences have problems with causality. At the level of a main process, we cannot sometimes determine the cause of events.

As a last note to the concept of emergence, we introduce here an unfortunate concurrence with the term "emergency."

In Section 2, we introduce references that deal with similar issues as our article. In Section 3, we briefly explain some theoretical concepts related to complex systems and emergences. Section 4 is devoted to a brief characterization of the physical approach for the modeling of complex systems. Section 5 deals especially with the conceptual background for structural invariants. In Section 6, the Emergent Situations (EMSs) analysis method is presented using a structural invariant (Matroid, Matroid Bases) (M, $\mathrm{MB})$ ) and a simple detection scheme (with $(M, \mathrm{MB})$ ) is introduced. The application of the developed method is illustrated in Section 7. Section 8 is devoted to the discussion of results. The method of tuning the quotient $(u / c)$ is introduced in Appendix A.

\section{Related Work}

In the next small overview, some works that could serve as an information as to how we approach the modeling of complex systems is introduced. These are main sources related to the topic of the article and ordered according to expert fields referred in the article.

2.1. Complex Systems and Emergent Situations. The basic concept of a complex system does not differ from the concept of the system - set of elements, bonds between them, and mutual interactions. However, a deeper analysis of complex systems discovers some peculiar properties that are very substantial for their identification and control (quasistability of state changes, nonlinear characteristics, self-organizing processes, etc.). The theory of complex systems is introduced in $[3,4,8,15]$ and modeling of complex systems is introduced in $[1,3,11,12]$. Literature sources [16-19] point out one approach of computational chemistry, which is helpful in understanding complex systems. Some fields of quantum physics, e.g., supergravity and supersymmetry, presently feature as problems of complex systems [20-22], especially problems of violation of supersymmetry in the role of the structural invariant. We cite from [21]: "The supersymmetry break cannot be done permanently by the particles of MSSM (Minimal Supersymmetric Standard Model) as they currently appear. This means that there is a new sector of the theory that is responsible for the breaking. The only constraint of this new sector is that it must break supersymmetry permanently and must give superparticles TeV scale masses."

2.2. Emergent Situations, Their Analysis, and Detection. Today's materialistic understanding of emergent phenomena continues to slip into the idea of a multilayered world in which we see only the upper layer. We fail to see what happens in the lower planes, and if the consequences of the phenomena from the lower planes penetrate to an upper layer, they seem inexplicable and emergent ones in the upper plane.

Without touching the relationship of causality and synchronicity (e.g., in the investigations of Jung, C. G., in the years 1913-1930, [23]), we value his opinion that the emergence is a creative process that is impossible to realize without the influence of a certain spiritual force [23, 24]. The problem is that these spiritual factors are hard to measure and outside the realm of physics. However, the concept of synchronicity has been investigated, e.g., as the collective behavior in the works of Herbert Haken [25], and lately in many works exploring the synchronization phenomena of large groups of living elements (fish in the ocean [26], birds in long way travels, and spontaneous light impulses of fire flies). And here, we are not sure if "collective behavior" leads to the synchronization phenomenon or if some other factor leads to the emergent phenomenon, "collective behavior." Then, the substance of emergence is not in a setup of "some inputs" but in synchronizing some events (processes). The synchronisation is possible to consider as an interaction between the local and global context in the complex system [27].

Besides Laughlin's examples of emergent phenomena in physics [13] (e.g., generation of particles as a consequence of breaking symmetry-Goldstone's theorem), there are known common examples which are very instructive and illustrate the nature of emergence phenomena, e.g., Kauffman's avalanche [28].

Very near to the original understanding of emergence is the approach of Reid [26] that is focused on emergent phenomena in biology. Reid collected a great amount of approaches and theories of emergence; however, they are oriented especially to emergent evolution. The importance of experiment in case of emergence is understood not only from a general point of view but also as a means for verification of the emergence result (not by the simulation). (The relation between emergence phenomena and simulation methods will be discussed in Section 3.) In recent years, works that use the concept of emergence in the solution of engineering problems were presented $[11,12]$. These works are associated with the possibility of simulation of the socalled actual emergent situations $\left(\mathrm{EMS}_{\mathrm{A}}\right)$.

Note 1. The true emergent situations $\left(\mathrm{EMS}_{\mathrm{T}}\right)$ are impossible to simulate. This fact contributes to the understanding of the analysis of emergent situations (resp., the detection of emergent situations). It will be discussed in detail in Section 3.1.

Besides, the introduced approaches include a large number of sources referring methods of artificial intelligence in connection with complex systems, especially with emergence phenomena in creative activities and problemsolving. From this field we quote only one source [29] in which are another references of the research line managed by John Gero. In last position we introduce our research of the analysis and the detection of emergent situations in complex systems $[5,10,15,30-32]$. 
2.3. Complex Systems as a Tool for Modeling of Evolution. For our proposed paper, is a very relevant works [14] (with clear thesis-"Emergence: half a quantum jump)"-and $[33,34]$ that attack the essence of causality and its contribution to predicting system development. The emergence is discussed in work [14] as a basic evolutionary operation (not as a preface for some disaster). It is associated with the concept of causality. In this article [33], the authors have investigated a large area of resources based on Granger's concept of causality and the concept of causality in dynamic systems. Finally, they focus on the analysis of paleontological time series and more specifically refer to three approaches:

\section{Stochastic differential equations (SDEs) \\ Convergent cross mapping (CCM) \\ Transfer of entropy (TE)}

In works $[33,34]$ is seen the effort not to go beyond the field of classical mathematics including the assumption about ordering and enumerating emergent phenomena. This assumption does not hold in our approach and therefore the mathematical tools used in our presented article is different from formal means used in $[33,34]$.

The mathematical background needed for our article is covered in [10, 32, 35-37]. The most important knowledge from these sources is a conceptual part of the theory of matroids combined with essential knowledge from Ramsey theory of graph.

\section{Complex Systems and Emergent Situations}

Complex systems have certain properties that differentiate them from other systems. The following features are examples of such properties that have already been classified, e.g., in $[3,4]$. (Another list of properties is introduced in [15])

Many elements in mutual interactions

Nondecomposability (irreducibility) into functional parts

Multidimensionality

Quasi-stability in state changes

Nonlinear characteristics

Self-organizing processes

Emergent behavior

Motion at the border of chaos

Nonstochastic future

Three most important features from the list above for the purposes of this article are as follows: elements in active interactions, self-organization, and emergent behavior (or emergent situations). In the next text, we remind certain conceptual background of emergent phenomena and of appearance of emergent situations (EMSs) (more examples in $[10,11,15,26,28,32,38])$.

Note 2. The large number of interacting elements is not a necessary property for the appearance of an emergent situation. Only in cases where we investigate a complex system with coincident events (e.g., in monitoring of many airplanes above the airport) we find them to be an essential condition. However, in many other cases, the emergent situation is because of other reasons.

A well-known and common example of the emergent situation brings the case of Kauffman's avalanche [28]: Put a pile of sand on the table. We observe the rolling of sand grains at the top of the cone. Suddenly, an avalanche breaks and slides to the base of the cone.

We do not know the reason of such an event or the time of further avalanche.

Of course, there are other types of emergent situations. Some of them appear suddenly, some of them for the first time, and some of them may appear only once.

Before we describe three types of EMSs, we try to emphasize few important circumstances that hold for all of them:

(A) Even if we know the causes of situations that can be simulated, there may be something that has not yet occurred and looks like emergence. This can be in generative systems. This will be referred to as the Actual Emergency Situation $\left(\mathrm{EMS}_{\mathrm{A}}\right)$. For a True Emergent Situation $\left(\mathrm{EMS}_{\mathrm{T}}\right)$, we do not know the causes.

(B) Emergence has no goal. If we do not know the goal of a phenomenon, it cannot be simulated. (It would be hard to check if the simulation has reached the correct goal.)

(C) True emergent situations are unpredictable ones.

(D) Describing emergence, we are still moving in an explicate order [39]. It means that we do not catch the true side of emergence (as it could be achieved using Implicate Order) and we are denied the true substance of emergence by the used semiotic factors and-above all-by the used language.

(E) Since some EMSs are seen (theoretically) for the first time and are unprepared, we cannot use the preprepared observational and symbolical tools as we know them from physics.

In this process, we must distinguish between the behavioral side and the symbolical side of the emergent phenomenon. (For example, we see the soliton in the water canal and we had not seen anything similar before. We capture its behavior (e.g., by a photograph) as a reflection of the natural phenomenon. Then, at home, we can find what solitons are (in books or in the Internet) and we use for their description corresponding equations.)

(F) In further text, we will distinguish two global levels of the EMS description.

Here, we denote the lowest phenomenological side of EMS as NAT and the symbolical side of the process as a level SYMB. We will further refine these descriptive levels in next sections.

3.1. Emergent Situations of Type A-Weak Emergent Situations. The causes of these situations and their output forms (outputs, shapes) are known. They can be recognized 
and their appearance can be predicted. Examples of processes and systems that generate such situations are: the Belousov-Zhabotinsky reaction, environments for initiating solitons, the oregonator, and the brusselator. They all belong also to the field of synergetics.

\subsection{Emergent Situations of Class B}

(b1) The causes of these situations are not known (in NAT), but their output forms are known.

(b2) The situation appears suddenly without an explicit association with situations in the previous relevant "time-space" context of the system.

(b3) In the mind of a human observer, the situation appears as a meaningful image situated in NAT, e.g., as a behavior (behavior of a group of termites), as a process (tsunami, traffic jam on the highway), and as a property (super conductivity).

(b4) It does not matter whether the EMS has been induced by the coincidence of events or by a sudden change in the complex system structure (in NAT) or by something else (SE). In all such cases, the EMS can be detected as a change of structure in the level of SYMB (e.g., the violation of structural invariant). However, it may be registered only as a "Possible Appearance of Emergent Situation (PAES)."

(b5) The detailed reasons and the internal causes of the appearance of $\mathrm{EMS}_{T}$ are not known (in NAT). Therefore, it is impossible to propose a complete prediction model of emergent situation but only a detection model for PAES (using structural invariant approach).

Situations belonging to this class include: a change in behavior strategy in a swarm colony; the appearance of floods $\left(\mathrm{EMS}_{A}\right.$ and $\left.\mathrm{EMS}_{T}\right)$; the appearance of rough waves in the ocean $\left(\mathrm{EMS}_{T}\right)$; and the appearance of traffic jams on the highway $\left(\mathrm{EMS}_{A}\right.$ and $\left.\mathrm{EMS}_{T}\right)$.

Note 3. Bifurcations of dynamic systems (sometimes also called phase transitions) are very close to the emergent situations of class B. Probably not all bifurcations (phase transitions), but cases of some attractors, e.g., the bifurcations of the Lorenz system, where the jumps between singular points show the characteristics of deteministic chaos, coincide with the understanding of emergent behavior and the global notion of structural invariant violation.

3.3. Emergent Situations of Class $C$. Such situations have the same properties as the situations from class B, except item $b 1$. Instead of it, we formulate condition $c 1$.

$c 1$. The causes of these situations are not known, and their output forms are also not known. No model of a situation of this kind is available before it first operates.

Situations that belong to this class include: potential instabilities in ecosystems $\left(\mathrm{EMS}_{T}\right)$; the appearance of artifacts in nano-structures $\left(\mathrm{EMS}_{T}\right)$; and the violation of supersymmetries in quantum mechanics $\left(\mathrm{EMS}_{T}\right)$.
For situations (B) and (C), we note that complex systems with emergences have the property of "weak causality" for some events. It is not possible to determine what is the result of what, and a logical consequence of such properties of $\left(\mathrm{EMS}_{T}\right)$ of types (B) and (C) is "If we do not know causes of situations it is impossible to simulate them."

Note 4. In order to distinguish between the simulation of processes and the analysis (detection) of emergent situations by a structural invariant approach, we introduce the following example: traffic jam on a highway.

For the simulation of this case using the physical approach, it is necessary to know causes (which we introduce in the simulation model as inputs or parameters). For example, someone "stepped on the brake," someone came unexpectedly from the left, someone installed a transport restriction, and somewhere the surface of the road has fallen down. And, it is necessary to simulate the unexpected situation, e.g., if we are $3 \mathrm{~km}$ in front of the place of such a change we encounter an unexplained stop. (This is a typical $\mathrm{EMS}_{A}$.) It is like with bifurcations. The physical description is causal, but there remains a part of the process that we cannot calculate (and simulate). It is not clear whether we have already calculated the traffic jam by adding some stochastic influence or not. In addition, there are certain causal activities that are needed to be done before adding a stochastic parameter. The problem is to decide on which variable "to hang" the noise on. In any case, we will only improve the causal model, which we must prove by experiment. The approach by structural invariant is another one: We quantify the performance of emergent phenomenon, i.e., the traffic jam. We calculate drivers, i.e., base and matroid, which are associated with the process on the highway. We do not know the causes of traffic jam, but we represent their associated portion by their "model," i.e., by adding traffic elements.

The situation will be investigated in more detail in the following section.

\section{A Physical Approach to Modeling of Complex Systems}

The physical approach is a result of fragmentation of the complex system as it corresponds to the explicate order [39]. Similarly, the description of the complex system is presented as a set of various relations between selected variables. The variables represent usually measurable quantities and the relations are formed into a closed system (enabling calculation of values of each variable by means of quantities of remaining variables). Such a description of a complex system has its background in physics. We recapitulate the essential properties of the physical description of a system:

Relations must be verifiable (usually through results and comparison with the experiment) 
Relations must be reproducible (they must be valid for reuse)

Relations must show causality (it must be clear what is causing what)

In physics and chemistry, these properties are included in a completely normal description-with the exception of emergent behavior. It is not clear for the systems we describe as macrostructures. Let us look at the example of the traffic jam in Figure 1.

Figure 1 is introduced here as a response to a natural question, "If the results from physical model are comparable with the results obtained by calculus for emergent situations."

The results of the physical model can only be compared with results from the calculation of emergent situations by converting physical model drivers to drivers of calculation of emergent situations. (Drivers are understood here as action quantities not men that drive cars.) So, the following described situation is rather exceptional: Let us say that we know only one common driver-the number of transport elements in interaction with each other, and that this number appears in the physical model as a parameter. In the block of calculation of emergent situations, we set the values of symptoms and calculate the basis of drivers. From the basis of drivers and from the condition for EMS, we calculate $\Delta V(\mathbf{B}, \mathbf{B}+1)$ as the number of drivers (number of transport elements), which must be added to the current state to induce a traffic jam. We add this number to the state of drivers in the physical model (i.e., we change the model parameters-dotted line) and compare the obtained new outputs (dotted line) of the physical model with the values of symptoms entering the block of calculation of emergent situations.

4.1. Physical Model. Examples of Inputs in the selected compartment of the highway: (i) "an average velocity of cars in a controlled segment of the highway," (ii) "weather changes (e.g., rain and fog)," (iii) "human influences," (iv) "changes in transport restrictions," and (v) "the quality of the highway surface (e.g., somewhere the road has disintegrated)."

Examples of Outputs in the selected compartment of the highway: (i) "an average number of cars within $1 \mathrm{~km}$," (ii) "visibility on the road," (iii) "number of bottlenecks (e.g., places of some repairs where it is allowed to use only one road strip) in $10 \mathrm{~km}$ of the road."

4.2. Calculation of Emergent Situations. Examples of some consequences associated with traffic jam in the selected segment of the highway are as follows:

Symptoms: (a) an average number of cars within $1 \mathrm{~km}$, (b) "visibility on the road," and (c) "number of bottlenecks (e.g., places of some repairs where is allowed to use only one road strip) in $10 \mathrm{~km}$ of the road."

Examples of Drivers associated with traffic jam are abstract drivers $\mathbf{B},(\mathbf{B}+1), \Delta V(\mathbf{B}, \mathbf{B}+1)$, and concrete drivers in our case-the number of transport elements added to actual state in the highway.

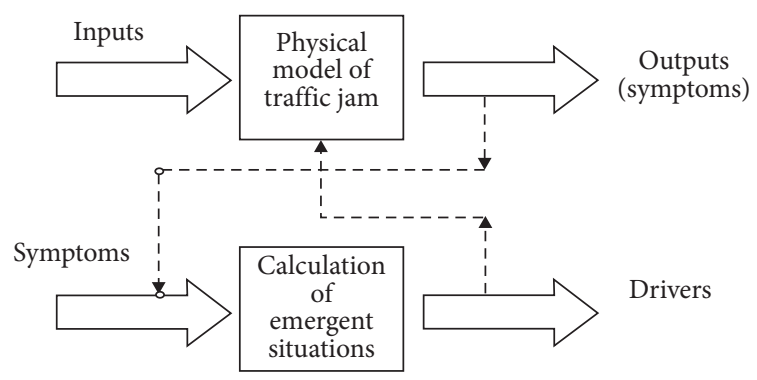

FIgURE 1: Comparison of the physical model of traffic jam with the block of calculation of emergent situations.

The block of calculation for emergent situations is not a model and it does not represent any causal relation. It is a "block" of calculation. After the change of parameters of the physical model (as a consequence of calculation of drivers), the physical model calculates the state after the emergent phenomenon-not the emergent phenomenon itself.

This brings us back to Laughlin's opinion to emergence and uncertainty, noting that the physical approach for describing complex systems does not capture emergent situations. It holds also for cases of large complex systems modelled by the physical approach, for example, in work [40] although supported by robust information technologies, e.g., in [41].

It probably does not make sense to extract and describe other individual models that belong to physical approach modeling or to create some similar others.

\section{Structural Invariant Approach to Modeling of Complex Systems}

5.1. Level of the Description in SYMB. The specificity of the description of complex systems depends on the description plane of the segment reality which is modelled. In terms of the general modeling theory, we refer to "local models," "compartments," or to "protomodels."

Let us consider a set of elements $\mathbf{C}$ of a compartment on a system and the set of bonds $\mathbf{S}$ (that interconnect elements) as the structure. (Bonds can represent interactions.)

Let us consider a set $\boldsymbol{\Omega}$ of automorphisms $m$ on the system $\langle C, S\rangle$ for which holds:

$$
\begin{aligned}
\forall x 1, x 2 & \in \mathbf{C}, \\
\forall b & \in \mathbf{S}, \\
\forall m & \in \mathbf{\Omega}, \\
m(x 1 b x 2) & =m(x 1) b m(x 2) .
\end{aligned}
$$

The pair $\langle\mathbf{C}, \mathbf{S}\rangle$ with a set of automorphisms $\Omega$ is the structural invariant. E.g., in the case of matroid, we have

$$
\begin{aligned}
M & =\left\langle X, \mathrm{IND},\left\{N_{1}, N_{2}, \ldots, N_{n}\right\}\right\rangle \\
& =\langle X, \mathbf{M B}\rangle \text { with the set } \Omega-\text { is the structural invariant, }
\end{aligned}
$$


where $X$ is a carrier of matroid; IND is a relation of independence; $N_{1}, N_{2}, \ldots, N_{n}$ are independent sets; the $\mathrm{MB}$ is a set of matroid bases; and $\Omega$ is the set of matroid automorphisms [38].

Similarly, for structural invariant "Hasse Diagram, Set of evaluated RULes" (denoted here as HD and RUL, respectively), we consider $\Omega$ as a set of lattice automorphisms.

5.2. Examples of Structural Invariants. We have discovered the following structural invariants. We have tested most of them, and we list them with the applications for which they were used. The introduced structural invariants are mostly algebraic in nature.

Examples of structural invariants:

Matroid, Bases of Matroid (M, BM). It is used for cases of floods, traffic jams, violation of the small water cycle, and desertification on Earth as in [5, 31, 32].

Dulmage-Mendelsohn Decomposition, Set of Evaluated RULes (DM, RUL). It is used in the detection of unexpected faults in thin-walled air space constructions [30].

Hasse Diagram, Set of Evaluated RULes (HD, RUL). It is used for the detection of emergent situations in the monitoring system [6].

Degree of Emergence, Interpretation Attractors (DE, $I A T)$. It is used for the discovery of novel artifacts in the conceptual design [42].

ALGebra of TRansformationS (ALG TRS). It is used for the detection of the onset of type 2 diabetes and cardiovascular diseases by analysis of ECG [43, 44].

Physical and geometrical SYMmetrieS (SYMS). It is used for the detection of EMS in some chemical processes [19]. It is known that spontaneous breaking of symmetry led to the formulation of the so-called electroweak force ((Glashow, Weinberg, Salam, 1967, 1979). Well-known Goldstone's theorem "particles necessarily emerge in any matter exhibiting spontaneous broken symmetry" is reminded also by Laughlin [13].

SUper SYsymmetry (SUSY) The phenomenon "LowScale SUSY Breaking" is described, for example, in [43] (Essential issues of SUper SYsymmetry have been discussed, e.g., in [20, 22]).

Violation of SI (in SYMB) is used for the detection of a Possible Appearance of Emergent Situation (PAES).

The ways of violation are special for each structural invariant and their generalization goes beyond the content of this article.

In the following sections, we will show how EMSs can be detected by a violation of structural invariant (M, BM).

\section{Analysis and Detection of EMSs by Structural Invariant (M, BM)}

6.1. Matroids and Ramsey Theory of Graph. Matroids are modern remarkable algebraic structures with very wide applicability. They have their typical representations, especially vector matroids and graph matroids. In terms of geometrical ideas-matroids belong to the so-called pregeometries.

From the matroid theory [35], we use the conceptual part and related concepts, as Independence Relation IND, basis, number of elements in the bases, etc., and several theorems that are in textbooks and which are not mentioned here. (However we implicitly work with them.)

Relations IND (Independence) and DN (Dependence) T represent not only algebraic relations between real or abstract elements (resp., between sets of elements) but they also "cover" real complex relations including interactions between elements.) The combination of matroid theory with Ramsey Theory of Graph (RTG) makes these disciplines a very powerful tool.

First, a few general facts are given.

A matroid is usually introduced [35] as the following structure:

$$
M=\left\langle X, \mathrm{IND},\left\{N_{1}, N_{2}, \ldots, N_{n}\right\}\right\rangle=\langle X, \mathbf{M B}\rangle,
$$

where $X$ is the ground set of elements (compartment); IND is a relation of independence; $N_{1}, N_{2}, \ldots, N_{n}$ are independent sets; and $\mathrm{MB}$ is a set of matroid bases. Matroid bases are maximum (according to cardinality) independent sets. In practical cases, it is advantageous to use a Dependence relation (DNT). (We consider only binary IND (DNT) relations in this article.) One of the possible Dependence (DNT) relations that respects problem factor $\alpha$ is the following one.

Definition 1. Elements $x_{1}, x_{2} \in X$ are Dependent (DNT( $x_{1}$, $\left.x_{2} \mid \alpha\right)$ ) with regard to a given problem factor $\alpha$ if one (or two, three, or four) of the following conditions holds (hold):

(i) Elements $x_{1}, x_{2}$ influence in a similar way in the compartment of a complex system with regard to a given problem factor $\alpha$ and taking into account elements from $X$ (expert criterion)

(ii) There are changes (variations) of $x_{1}$ which are associated with changes (variations) in $x_{2}$ (or $v v$ ), with regard to a given problem factor $\alpha$ and with regard to elements from $X$

(iii) The application of $x_{1}$ implies the application of $x_{2}$ (or $v v$ ), with regard to a given problem factor $\alpha$ and with regard to elements from $X$

(iv) The application of $x_{1}$ excludes the application of $x_{2}$ (or $v v$ ), with regard to a given problem factor $\alpha$ and with regard to elements from $X$

Examples of the problem factor: phenomenon of floods, fault in the system, and increase of temperature.

Note 5. There are of course other relations of Dependence [35]. A general view to the number of possible Dependence relations is introduced in [45].

For our conditions, a simple theorem holds. 


\section{Theorem 1}

$$
\forall x_{1}, x_{2} \in X,\left(\operatorname{not}\left(\operatorname{DNT}\left(x_{1}, x_{2} \mid \alpha\right)\right)\right) \Leftrightarrow\left(\operatorname{IND}\left(x_{1}, x_{2} \mid \alpha\right)\right) .
$$

Before we formulate an essential rule for the detection of an emergent situation, we describe the construction of a matroid and its bases on a set $X$.

Construction. Matroid is formed in a squared matrix $[(\# X) \times(\# X)]$. Matroid is formed by an expert who responds to the question: "Are elements $x_{i}, x_{j}$ dependent with respect to problem factor $\alpha$ (according to definition Def.1.) $\operatorname{DNT}(x 1, x 2 \mid \alpha))$ ?" If yes, the corresponding field of matrix is fulfilled by "1." If not, by "0." If the question is nonrelevant, the field will be "*." According to Theorem 1, a basis of matroid is represented by elements of $X$, for which it holds

$$
\begin{aligned}
\mathbf{B} & \subseteq X, \\
\# X & =n, \\
\mathbf{B} & =\left\{x_{i 1}, \ldots, x_{i m}\right\} \mid \forall\left(x_{i j}, x_{i k}\right) \operatorname{DNT}\left(x_{i j}, x_{i k} \mid \alpha\right)=0, \\
& \text { for all } j, k=1, \ldots, m .
\end{aligned}
$$

\section{Theorem 2}

$$
\forall \mathbf{B}_{k}, \ldots, \mathbf{B}_{m} \in \mathbf{M B}, \quad \# \mathbf{B}_{k}=\ldots,=\# \mathbf{B}_{m} .
$$

All bases of the matroid have the same number of elements.

Now, we may form an essential sentence for the analysis (detection) of the appearance of an emergent situation:

An extension of a matroid basis by at least one element is considered (in this article) as a violation of structural in$\operatorname{variant}(M, \mathrm{BM})$, and it is considered as an internal indicator of the appearance of an emergent situation.

6.1.1. Extension of Matroid Basis. It is clear that the extending element must come from outside the original matroid. In abstract space where is situated considered matroid it is enough to verify the relation IND. However, turning back to the reality of the emergent phenomenon this "abstract" element must be semantically associated with the real element of the external description. (in detail, Section 6.3 provides description of "normalization")

The extension of a basis of the matroid can be done in two ways:

(m1) From the cardinality of the matroid, we calculate the minimum number of elements that must be added to the matroid to have an extending element among them-discussed in detail in Section 6.3. The number " $\min \Delta f(R N)$ " is a minimal difference between the next Ramsey number and the current Ramsey number (Section 6.3, expression (16)).

(m2) We find an extending element, verify the possibility of the extension of some basis (IND), and if it is necessary we make normalization (explanation in Section 7.3).

6.1.2. Association with Ramsey Theory of Graph [37]. Speaking about graph matroids, we consider many possible graphic architectures in [35]. Relations DNT and IND are considered as binary relations and they are represented by edges with connected and disconnected nodes in graphs. In the following text, we work with matroids that are formed on perfect graphs and the following consequences can be used.

The bases (MB) will be constructed as perfect subgraphs in a perfect graph on $X$. (A perfect graph on $X$ has each node connected with all other nodes of $X$.)

The independent and dependent elements in a perfect graph $G_{p}=(V, E)$ are easily constructed by coloring the edges of the perfect graph by two colors, and the formalism of Ramsey numbers $-R(\# \mathbf{B}, \# Y), \mathbf{B} \in \mathrm{MB}$ is offered for use. We now introduce a free formulation of the relations on a perfect graph $G_{p}$ colored by two colors as a consequence of the famous Ramsey theorem.

Theorem 3. A perfect graph $G_{p}=(V, E)$ with $n$ nodes, where each edge belongs to class $A$ or class $B$, contains a perfect subgraph with a nodes and edges from class $A$ or a perfect subgraph with $b$ nodes and edges from class $B$.

In most cases holds $(\# \mathbf{B}+\# Y) \neq n$ for $R(\# \mathbf{B}, \# Y)$, $\mathbf{B} \in \mathrm{MB}$. The reason is that remaining nodes $(n-\# \mathbf{B}-\# Y)$ belong to class A or class B; however, they do not form perfect subgraphs.

Number $R(a, b)=n$ that corresponds to numbers " $a$ " and " $b$ " and is equal to the number of nodes of the perfect graph $G_{p}$ is called a Ramsey number.

Note 6 (historical note). Until now, only some Ramsey numbers (RNs) are known, e.g., $R(3,3)=6, R(3,4)=9, R(3$, 5) $=14, R(3,6)=18, . ., R(3,15)=[73,78], \ldots, R(4,4)=18$, $R(4,11)=[96,191], \ldots, R(6,10)=[177,1171], \ldots, R(10$, $10)=[798,23556], \ldots$, and $R(19,19) \geq 17885$. The brackets [., .] denote intervals of integer numbers. (Known quantities from the table in [37] will be used for computations in Section 7).

6.1.3. The "Anonymity" of Ramsey Theorem. Note that Ramsey theorem responds to the question "how many" (elements) and not "which" (elements). For our case, it means that we are able to compute how many elements are necessary to add to the original matroid for the extension of a basis; however, we do not know which elements are necessary to add.

6.2. Power and Complexity of an Emergent Phenomenon. The emergent phenomenon we describe, in our view, by two variables, the power $H_{P}$ of the emergent phenomenon and the complexity $H_{\mathrm{COM}}$ of the emergent phenomenon.

Essential relations between the power $H_{P}$ and the complexity $H_{\mathrm{COM}}$ are expressed by the following two equations: 


$$
\begin{gathered}
H_{P}(\mathbf{B}+1)=H_{P}(\mathbf{B})+\left(\frac{u}{c}\right) H_{\mathrm{COM}}(\mathbf{B}), \\
H_{\mathrm{COM}}(\mathbf{B}+1)=H_{\mathrm{COM}}(\mathbf{B})+u H_{P}(\mathbf{B}),
\end{gathered}
$$

where $\mathbf{B} \in \mathrm{MB}$ is the basis of a matroid and $\mathbf{B}+1$ is the basis $\mathbf{B}$ extended by one element. Variables $H_{P}(\mathbf{B}), H_{P}(\mathbf{B}+1)$, and $H_{\mathrm{COM}}(\mathbf{B}), H_{\mathrm{COM}}(\mathbf{B}+1)$ are power and complexity of the emergent phenomenon related to bases $\mathbf{B}$ and $\mathbf{B}+1$.

Symbol $u$ denotes the quotient of self-organization of the considered complex system (represented by the compartment) and $c$ is the limit of this quotient (the best self-organization). Quotient $(u / c) \in\langle 0,1\rangle$ represents "intelligence" of the self-organizing process that will execute the emergent phenomenon. Operating with equation (8), we obtain the contribution to power released by the emergent phenomenon:

$$
\Delta H_{P}(\mathbf{B}+1)=\left(\frac{u}{c}\right) H_{\mathrm{COM}}(\mathbf{B}),
$$

where $\Delta H_{P}(\mathbf{B}+1)$ represents the registrable changes in the system due to EMS and $H_{\mathrm{COM}}(\mathbf{B})$ represents the complexity of the system of drivers that provide $\Delta H_{P}(\mathbf{B}+1)$.

The link between $\Delta H_{P}(\mathbf{B}+1)$ and $H_{\mathrm{COM}}(\mathbf{B})$ is provided by $(u / c)$-the "intelligence" quotient of the system. The quantity of $(u / c)$ is done by rough estimations in and by soft tuning in Appendix A.

In works $[31,32]$ were introduced orientation quantities for quotients $(u / c)$, as given in Table 1 .

As will be seen in Section7, the value of $(u / c)$ is very sensitive and important for the result of the application of expression (9). The values introduced in Table 1 are characteristic values for the corresponding classes of complex systems but not absolutely rigged for computation. For example, the value of $(u / c)$ for the first class of complex systems ("inanimate" natural systems-avalanches, deserts, floods, earthquake, tsunami) may alternate between 0.06 and 0.15 .

The contribution to the power of the emergent phenomenon $\Delta H_{P}(\mathbf{B}+1)$ that has an intuitive meaning in the internal level is associated with the external level of the description with the power $\Delta H_{D}(\mathbf{B}+1)$ (e.g., damage of houses by floods) measured by quantities of external variables (symptoms) $s_{i}, i=1, \ldots, n$ for emergent $\left(s_{\text {iem }}\right)$ and for nominal $\left(s_{\text {inom }}\right)$ situations:

$$
\begin{aligned}
& \Delta H_{D}(\mathbf{B}+1)=\left(\sum_{i=1, n}\left(\omega_{i}\left(\frac{s_{\text {iem }}}{s_{\text {inom }}}\right)^{2}\right)\right)^{(1 \backslash 2)}, \text { for } i=1, \ldots, n, \\
& \Delta H_{P}(\mathbf{B}+1)=\Lambda \cdot \Delta H_{D}(\mathbf{B}+1),
\end{aligned}
$$

TABLe 1: Orientation values of $(u / c)$.

\begin{tabular}{lc}
\hline$(u / c)$ & $\begin{array}{c}\text { Classes of complex systems-the examples of complex } \\
\text { systems }\end{array}$ \\
\hline 0.1 & "Inanimate" natural systems-avalanches, systems of \\
clouds, floods, earthquake, tsunami \\
$0.2-0.4$ & $\begin{array}{c}\text { Complex systems in chemical and nuclear applications } \\
\text { Lower alive systems-bees, termites, ants }\end{array}$ \\
$0.3-0.4$ & $\begin{array}{r}\text { Mixed natural and artificial systems-ecological systems, } \\
\text { social systems, transport systems }\end{array}$ \\
0.5 & $\begin{array}{r}\text { Large complex systems in the ocean (huge shoals of fish) } \\
\text { and in atmosphere (shoals of birds) }\end{array}$ \\
$0.6-0.8$ & $\begin{array}{r}\text { Advanced organism systems-human brain, metabolic } \\
\text { systems, biochemical systems }\end{array}$ \\
0.9 & $\begin{array}{r}\text { Metaphorical systems-human mind, systems with high } \\
\text { concentration of information } \\
1\end{array}$
\end{tabular}

where $\omega_{i}$ are quotients of importance and $\Lambda$ is a calibration constant.

Quotients of importance $\omega_{i}$ are computed by using the Saaty method [46].

The contribution to power of the emergent phenomenon $\Delta H_{D}(\mathbf{B}+1)$ results in a dimensionless real number expressed herein as \% (e.g., contribution for $20 \%$ is calculated as (120/ $100)=1.2$ ). Equation (8) is associated with equation (7) where $H_{\mathrm{COM}}($.$) is approximated in our case by the number of$ elements of basis B, i.e. \#B. Function $\xi$ transforms real number $\left(\Delta H_{p}(\mathbf{B}+1)\right) /(u / c)$ into the nearest larger integer and holds:

$$
\xi\left(\frac{\Delta H_{P}(\mathbf{B}+1)}{(u / c)}\right)=\# \mathbf{B} .
$$

For explanation, we describe a contribution $\Delta \mathrm{H}_{\mathrm{D}}(\mathrm{B}+1)$ of power of a possible EMS by approximating the emergent and nominal values of the selected variables in the compartment of real world. Using calibration constant $\Lambda$, we find $\Delta H_{P}(\mathbf{B}+1)$. Then, we form a corresponding representation in the pregeometry (matroid and matroid bases) and we compute by (12) the cardinality \# B . We solve the violation of this invariant as an extension of a matroid basis (by ways $m 1$ or $m 2$-Section 6.1). Then, if necessary, conditions for the proposed EMS are computed in the original compartment of the real world-called herein "normalization"-Section 6.4.)

6.3. A Simple Scheme for the Analysis (Detection) of an Emergent Situation. The description of the processing emergent situation that we want to indicate in the complex system compartment has the following form in NAT and SYMB:

NAT: (synchronization of events) or (change of the structure of CS) or S.E. $\longrightarrow$ PAES,

$$
\text { SYMB: } \Delta H_{P}(\mathbf{B}+1) \longrightarrow \text { structural invariant }(\mathrm{SI}) \longrightarrow \text { VSI } \longrightarrow \text { detection of PAES, }
$$


where S.E. symbolizes "Something Else," PAES is a "Possible Appearance of Emergent Situation," SI is a structural invariant, and VSI is a Violation of Structural Invariant, and $\Delta H_{P}(\mathbf{B}+1)$ is a contribution of power of the emergent phenomenon in SYMB.

In case SI is a matroid and its bases, the following expression holds:

$$
\begin{aligned}
\text { SYMB: } \Delta H_{P}(\mathbf{B}+1) & \longrightarrow\langle X 1, \mathbf{M B} 1\rangle \longrightarrow \\
\langle X 1, \mathbf{B} 1\rangle & \longrightarrow\langle X 1 \cup E 1, \mathbf{B} 1 \cup e 1\rangle \longrightarrow\langle X 2, \mathbf{B} 2\rangle,
\end{aligned}
$$

where $X 1, X 2$ are carriers of matroids, $\mathbf{M B} 1$ is a set of bases on $X 1, \mathbf{B} 1$ is a basis from $\mathrm{MB} 1, E 1$ is a set of elements that extends $X 1, e 1$ is an element that extends basis $\mathbf{B} 1$, and $\mathbf{B} 2$ is a basis on $X 2$.

Note 7. Expressions (14) and (15) describe operations associated with pregeometry (matroid).

Example 1. $\# X=1600$. ( $\# \mathbf{B}=11$ for $\# X \geq 1597)$. The "threat" of PAES (in NAT) is "represented" (in SYMB) with the addition of at least 40 elements that enable one element extension of the Basis ( $\# \mathbf{B}=12$ for $\# X \geq 1637$ ).

We formulate now a basic rule for analyzing (detecting) an emergent situation (in relation with expression (14)) as follows.

For case $m 1$ in Section 6.1,

$$
\operatorname{IF}(\# E 1 \geq \min \Delta f(\mathrm{RN})) \Rightarrow(\mathrm{PAES}),
$$

where $E 1$ is a set that extends the matroid $\langle X 1, \mathrm{MB} 1\rangle$ (15) and contains at least one element $e 1$ extending basis $\mathbf{B} 1$. The number "min $\Delta f(R N)$ " is the minimal difference between the next Ramsey number and the current Ramsey number, and PAES denotes the "Possible Appearance of an Emergent Situation.”

The number $\Delta f(\mathrm{RN})$ provides that at least one element could be connected with relation IND with all elements of some basis from MB1. (This is the case for \# B not for \#Y-in the formalism of Ramsey numbers $R(\# \mathbf{B}, \# Y)$-Theorem 3.)

For the case $m 2$,

$$
\operatorname{IF}\left(\exists e,\left(\operatorname{IND}\left(e, x_{i}\right), \quad \forall x_{i} \in \mathbf{B} 1\right)\right) \Rightarrow(\mathrm{PAES}),
$$

where " $e$ " is an extending element, for which there was verified Independence (IND) to all elements of $\mathbf{B} 1$.

6.4. Normalization of $\Delta f(R N)$ with regard to a Real "Volume" of Added Elements. The following operation is actual for cases where the detecting of PAES in pregeometry can be represented by the addition of real elements to the real compartment (e.g., drivers in floods) or the addition of some time interval to the given time moment [5] in the considered compartment. (We are speaking about an additive representation of drivers.)

Equations (9), (16), and (17) introduce a method for the detection of PAES without considering the actual state of the process (for which we compute the emergent phenomenon).
The actual state corresponds to some number of active elements of the process that are associated with the emergent phenomenon (e.g., transport elements (cars) in case of traffic jam). This number $V(\mathbf{B})$ is different from $\mathrm{RN}(\mathbf{B})$-in general. Then, it is necessary to execute a normalization of $\Delta_{j} f(\mathrm{RN})$ to the actual number of active elements $V(\mathbf{B})$ in the actual state of a complex system. The information about number $V(\mathbf{B})$ is a complementary information, and it does not influence the calculation according to equations (9), (16), and (17). The normalization procedure for the alternative " $m 1$ " (expression (16)) has the following steps:

Let us assume that we have computed $\Delta H_{P}(\mathbf{B}+1)$ (equations (10) and (11)).

From this number, it is possible to acquire \#B by equation (12).

Considering \# $\mathbf{B}$ as a number of elements of a basis, we compute $\mathrm{RN}(\mathbf{B})$.

This number corresponds to an actual state of the complex system and thus to a "volume" of elements in this state.

Let us denote: $\mathrm{RN}(\mathbf{B})$ corresponds to $V(\mathbf{B})$.

From this correspondence, we extract a normalization quotient $\lambda$ :

$$
\lambda=\frac{V(\mathbf{B})}{\mathrm{RN}(\mathbf{B})} .
$$

After computation of $\mathrm{RN}(\mathbf{B}+1)$, we extrapolate the quantity $V(\mathbf{B}+1)$

$$
V(\mathbf{B}+1)=\lambda \mathrm{RN}(\mathbf{B}+1) .
$$

Then, the value $\Delta f(\mathrm{RN})$ corresponds to value $\Delta V(\mathbf{B}, \mathbf{B}+1)$ (the number of elements that is necessary to add to $V(\mathbf{B}))$.

$$
\Delta V(\mathbf{B}, \mathbf{B}+1)=\lambda \Delta f(\mathrm{RN}) .
$$

And, the addition of $\Delta V(\mathbf{B}, \mathbf{B}+1)$ to the number of active elements of the actual state (in SYMB) is associated with PAES (in NAT).

For the case " $m 2$ " (expression (17)),

$$
\lambda=\frac{V(\mathbf{B})}{\mathrm{RN}(\mathbf{B})} .
$$

After computation of $\mathrm{RN}(\mathbf{B}+1)$, we extrapolate the quantity $V(\mathbf{B}+1)$ :

$$
\begin{aligned}
V(\mathbf{B}+1) & =\lambda(\mathrm{RN}(\mathbf{B})+1) \\
& =\left(\frac{V(\mathbf{B})}{\operatorname{RN}(\mathbf{B})}\right)(\mathrm{RN}(\mathbf{B})+1)=V(\mathbf{B})+\lambda .
\end{aligned}
$$

Then, the value $\Delta f(\mathrm{RN})$ corresponds to value $\Delta V(\mathbf{B}, \mathbf{B}+1)$ (the number of elements that is necessary to add to $V(\mathbf{B}))$. 


$$
\Delta V(\mathbf{B}, \mathbf{B}+1)=\lambda .
$$

Summary of the whole process of analysis is given in Figure 2.

In Figure 2 is illustrated the following process: in the Model of Reality are estimated possible values of symptoms $\left(s_{\mathrm{em}},\left(\mathrm{s}_{\text {nom }}\right)\right)$ that represent the considered emergent phenomenon. By means of these quantities, $\Delta H_{D}(\mathbf{B}+1)$ and then $\Delta H_{P}(\mathbf{B}+1)$ are computed. This value is transformed into the space of pregeometry as a matroid and its bases. These structures represent drivers for the emergent phenomenon. By the normalization procedure (for alternatives " $m 1$ " or " $m 2$ ") the values of complements $\Delta V(\mathbf{B}, \mathbf{B}+1)$ are acquired that could be added to the actual state in the Model of Reality as a condition for a Possible Appearance of Emergent Situation (PAES).

\section{Application of the Developed Method on Modeling the Complex System and the Analysis of EMSs by means of $\langle M, B M\rangle$}

In this section are presented examples of modeling complex systems, especially of the analysis and the detection of emergent situations. Examples lead to the discovery of "Conditions for indicating PAES."

The first two cases are rather methodological ones, i.e., they show how to calculate emergences. They are not bound to any specific data and they are not used for comparison with an experiment. The onset and consequences of these EMSs in everyday life are generally known. The third example is tied to specific data and may be compared with results of real situations.

7.1. Floods. In this section, we do not want to compete with very solid studies of heavy rainfall events that have been conducted around the world in the context of climate changes. At least two works $[47,48]$ were devoted to the study of rainfall events in the Czech Republic, which very systematically compared with the outputs achieved by simulations in Regional Climate Models (RCM) against the observation data "at site." Although heavy rainfall events have not been identified directly as the cause of floods, we do get some small satisfaction from the conclusion of article [48] in relation to our view of the difficult simulability of such events: "The RCMs on average represent the number of heavy rainfall events, seasonal total precipitation due to heavy events and event depths relatively well; however, the number of heavy events as well as the corresponding seasonal totals are overestimated at higherelevated grid boxes."

Floods belong to emergent situations. Especially, socalled sudden (flash) floods with onset in hours or sometimes in minutes. From the point of view of an lay person, they are induced by heavy rains and by aggregated volumes of water. On a deeper investigation, we may observe that there are further influencing factors [49], as follows:
Self-organizing process which interacts with water carriers such as rivers, brooks, streams, and ponds

Changes in the internal structure of the hydrological system (e.g., ruptured barriers of water dams and ponds)

Something else (S.E.) (invisible)

There are many variables that characterize the individual factors mentioned above, and we do not know how they divide their influence. In addition, it is impossible to describe the mutual interaction of the acting elements-for example, by multiagent formalism. But, even there we could not identify which of the influences is causing the floods. For this reason, we work with integral values, which we convert to pregeometry. From this, we then calculate the "scalar" increment of elements (rivers, streams, etc.). But, we cannot claim that this contribution will cause floods, we only claim that it is associated with them.

The "self-organizing" process is hard to describe but facilitates the calculation of conditions of floods in a compartment. (Compartment is considered here as a selected part of the landscape that is strongly influenced by the floods.)

The calculus used for the solution of the problem was explained in Section 6:

According to the possible variables in the external description, we consider floods as a two-dimensional case

In equation (11), we consider the calibration constant $\Lambda=1$ and thus $\Delta H_{P}(\mathbf{B}+1)=\Delta H_{D}(\mathbf{B}+1)$

Two external variables are used for the computation of $\Delta H_{P}(\mathbf{B}+1):$

$x 1$ : an average height of water levels in water carriers (rivers, brooks, streams, basins, etc. that interact in the considered region)

$x 2$ : number of buildings in danger of flooding, in the considered region

The following nominal and emergent quantities are considered:

$x 1_{\text {nom }}=3 \mathrm{~m}$ and $x 1_{\mathrm{em}}=8.1 \mathrm{~m}$. The quotient of importance $\omega_{1}=0.4$.

$x 2_{\text {nom }}=1000$ and $x 2_{\mathrm{em}}=2500$. The quotient of importance $\omega_{2}=0.6$.

Applying expressions (10) and (11) (for $\Lambda=1$ ), we compute

$$
\begin{aligned}
\Delta H_{P}(\mathbf{B}+1) & =\left(\left(\omega_{1}\left(\frac{x_{1 \mathrm{em}}}{x_{1 \mathrm{nom}}}\right)\right)^{2}+\left(\omega_{2}\left(\frac{x_{2 \mathrm{em}}}{x_{2 \mathrm{nom}}}\right)\right)^{2}\right)^{1 / 2} \\
& =\left((0.4 * 2.7)^{2}+(0.6 * 2.5)^{2}\right)^{1 / 2}=1.85 .
\end{aligned}
$$
A.
Now, we use the method for tuning $(u / c)$ from Appendix 


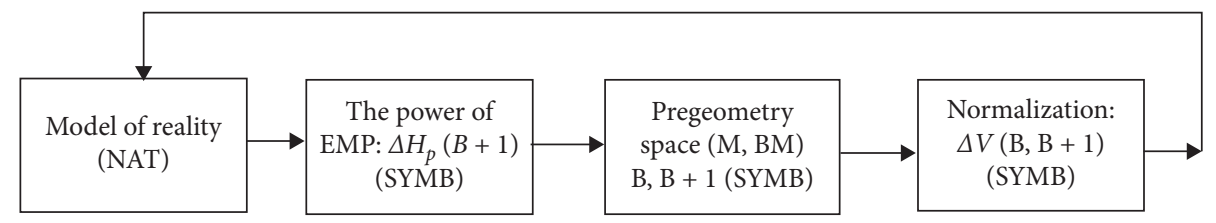

Figure 2: The analysis of EMSs.

For the first estimation of $(u / c)_{0}$, we set up $(u / c)_{0}=0.09$ (from Table 1).

Using expression (9), we compute the first approximation of cardinality of the basis \#B:

$$
H_{\mathrm{COM}}(\mathbf{B})=\xi\left(\frac{\Delta H_{P}(\mathbf{B}+1)}{(u / c)_{0}}\right)=\xi\left(\frac{1.85}{0.09}\right)=21=\# \mathbf{B}
$$

For $\# \mathbf{B}=21$, we find in tables [37] Ramsey numbers $R(\# \mathbf{B}, Y): R(21,3), R(21,4), R(21,5), R(21,6), R(21,7), R(21$, 8): $\{R(21,3) \geq 122, R(21,4) \geq 242, R(21,5) \leq 10626, R(21$, $6) \leq 53130, R(21,7) \geq 1214$, and $R(21,8) \geq 1328\}$.

For $\#(\mathbf{B}+1)=22$, we find in tables [37] Ramsey numbers $R(\# \mathbf{B}+1, Y):\{R(22,3) \geq 125, R(22,4) \geq 282, R(22$, $5) \geq 422, R(22,6) \geq 1070, R(22,7) \leq 296010$, and $R(22,8) \leq 1$ $184040\}$.

We are looking for a minimum difference: $\Delta_{0} f(\mathrm{RN})=$ $\min \{(125-122),(282-242)$, smaller differences do not exist $\}$,

$$
\begin{aligned}
\Delta_{0} \mathrm{f}(\mathrm{RN}) & =3 \\
\operatorname{for}\left(\frac{u}{c}\right)_{1} & =0.1 \\
H_{\mathrm{COM}}(\mathbf{B}) & =\xi\left(\frac{\Delta H_{P}(\mathbf{B}+1)}{(u / c)_{1}}\right)=\xi\left(\frac{1.85}{0.1}\right)=19=\# \mathbf{B} .
\end{aligned}
$$

For $\# \mathbf{B}=19$, we find in tables [37] Ramsey numbers $R(\# \mathbf{B}, Y): R(19,3) \geq 106, R(19,4) \geq 198, R(19,5) \geq 338, R(19$, $6) \geq 710, R(19,7) \geq 908$, and $R(19,8) \geq 1054$.

For $\#(\mathbf{B}+1)=20$, we find in tables [37] Ramsey numbers $R(\# \mathbf{B}+1, Y):\{R(20,3) \geq 109, R(20,4) \geq 230, R(20$, $5) \geq 380, R(20,6) \geq 878, R(20,7) \leq 296010$, and $R(20,8) \geq$ 1094.

We are looking for a minimum difference: $\Delta_{1} f(\mathrm{RN})=$ $\min \{(109-106),(230-198),(380-338),(878-710)$, (1094-1054), smaller differences do not exist $\}$ :

$$
\begin{aligned}
\Delta_{1} f(\mathrm{RN}) & =3 \\
\text { for }\left(\frac{u}{c}\right)_{2} & =0.15 \\
H_{\mathrm{COM}}(\mathbf{B}) & =\xi\left(\frac{\Delta H_{P}(\mathbf{B}+1)}{(u / c)_{2}}\right)=\xi\left(\frac{1.85}{0.15}\right)=13=\# \mathbf{B} .
\end{aligned}
$$

For $\# \mathbf{B}=13$, we find in tables [37] Ramsey numbers $R(\# \mathbf{B}, Y): R(13,3)=[59,69], R(13,4)=[133,291], R(13,5) \geq$ $193, R(13,6)=278, R(13,7) \geq 511$, and $R(13,8) \geq 635$.

For $\#(\mathbf{B}+1)=14$, we find in tables [37] Ramsey numbers $R(\# \mathbf{B}+1, Y):\{R(14,3)=[66,78], R(14,4)=[141$, 349], $R(14,5) \geq 221, R(14,6) \geq 292, R(14,7) \leq 296010$, and $R(14,8) \leq 1184040\}$.

We are looking for a minimum difference: $\Delta_{2} f(\mathrm{RN})=$ $\min \quad\{(66-59), \quad(78-69), \quad(141-133), \quad(221-193)$, (292-278), smaller differences do not exist $\}$ :

$$
\begin{aligned}
\Delta_{2} f(\mathrm{RN}) & =7, \\
\text { for }\left(\frac{u}{c}\right)_{3} & =0.2, \\
H_{\mathrm{COM}}(\mathbf{B}) & =\xi\left(\frac{\Delta H_{P}(\mathbf{B}+1)}{(u / c)_{3}}\right)=\xi\left(\frac{1.85}{0.2}\right)=10=\# \mathbf{B} .
\end{aligned}
$$

For $\# \mathbf{B}=10$, we find in tables [37] Ramsey numbers $R(\# \mathbf{B}, Y): R(10,3)=[40,43], R(10,4)=[80,149], R(10,5) \geq$ $[141,442], R(10,6)=[177,1171], R(10,7)=[1,2826]$, and $R(10,8)[316,6090]$.

For $\#(\mathbf{B}+1)=11$, we find in tables [37] Ramsey numbers $R(\# \mathbf{B}+1, Y):\{R(11,3)=[46,51], R(11,4)=[96$, $191], R(11,5) \geq 153, R(11,6) \geq 253, R(11,7) \geq 322$, and $R(11$, 8) $\leq 1184040\}$.

We are looking for a minimum difference: $\Delta_{3} f(\mathrm{RN})=$ $\min \{(46-40),(46-43),(51-43),(96-80),(153-141)$, smaller differences do not exist $\}$ :

$$
\begin{aligned}
\Delta_{3} f(\mathrm{RN}) & =3 \\
\text { for }\left(\frac{u}{c}\right)_{4} & =0.08 \\
H_{\mathrm{COM}}(\mathbf{B}) & =\xi\left(\frac{\Delta H_{P}(\mathbf{B}+1)}{(u / c)_{4}}\right)=\xi\left(\frac{1.85}{0.2}\right)=24=\# \mathbf{B} .
\end{aligned}
$$

For \# $\mathbf{B}=24$, we have only approximate estimates of RNs in the form of inequalities.

The sequence of values leads to $\min \left(\Delta_{0} f\left(\mathrm{RN} \Delta_{3} f(\mathrm{RN})\right)=\right.$ $\min (3,3,7,3)=3$.

So, it is possible to work with $(u / c)=0.09$ or 0.1 .

We turn back to $(u / c)=0.09$ :

$$
H_{\mathrm{COM}}(\mathbf{B})=\xi\left(\frac{\Delta H_{P}(\mathbf{B}+1)}{(u / c)_{0}}\right)=\xi\left(\frac{1.85}{0.09}\right)=21=\# \mathbf{B} .
$$


For $\# \mathbf{B}=21$, we find in tables [37] Ramsey numbers $R(\# \mathbf{B}, Y): R(21,3), R(21,4), R(21,5), R(21,6), R(21$, $7), R(21,8):\{R(21,3) \geq 122, R(21,4) \geq 242, R(21,5) \leq 10626$, $R(21,6) \leq 53130, R(21,7) \geq 1214$, and $R(21,8) \geq 1328\}$.

For $\#(\mathbf{B}+1)=22$, we find in tables [37] Ramsey numbers $R(\# \mathbf{B}+1, Y):\{R(22,3) \geq 125, R(22,4) \geq 282, R(22$, $5) \geq 422, R(22,6) \geq 1070, R(22,7) \leq 296010$, and $R(22,8) \leq 1$ $184040\}$.

Optimal pairs of RNs are $\Delta_{\text {opt }} f(\mathrm{RN})=(125-122)$ OR $(282-242)\}=3$ OR 40 .

7.1.1. Normalization of $\Delta_{\text {opt }} f(\mathrm{RN})$

The additional information about the number of active elements in the actual state of the complex system is $V(B)=$ 300 water carriers (streams, lakes, ponds, etc.).

For $\mathrm{RN}(B)=\mathrm{R}(21,3)=122$ and $\lambda=V(B) / \mathrm{RN}(B)$, we calculate $\lambda=2.46$.

According to (15) and (18) and for $\Delta_{\text {opt }} f(\mathrm{RN})=$ $(125-122)$ OR $(282-242)$, we have $\Delta V(\mathbf{B}+1)=8$ OR 98.

Note 8. More real is the second case $-\Delta V(\mathbf{B}+1)=98$.

Note 9. .The computation of other optimal pairs of Ramsey numbers was complicated in this case by small knowledge of precise values of Ramsey numbers, e.g., $(R(21,5) \leq 10626$, $R(21,6) \leq 53130), R(22,7) \leq 34337716$.

The answer to the question about flood conditions is: The critical contribution of water carriers that is associated with the assumed power $\Delta H_{P}(\mathbf{B}+1)$ is 98 (for the actual state$=300$ water carriers). This number only approximates the EMS and "covers" both sudden changes in the structure of the hydrological system and also something else (invisible).

7.2. Traffic Jam on the Highway. In work [50] is confirmed that traffic jam on the highway is a process with chaos and thus is hard to detect it. In order to distinguish between $\mathrm{EMS}_{\mathrm{A}}$ and $\mathrm{EMS}_{\mathrm{T}}$, a brief discussion has been introduced in Section 3, and we remind it here.
For simulation of the traffic jam process using the physical approach, it is necessary to know causes. e.g., someone "stepped on the brake," someone came unexpectedly from the left, someone installed a transport restriction, somewhere the surface of the road has deteriorated. If we are $3 \mathrm{~km}$ ahead of the place of such a change, we encounter an unexplained stop. (This is a typical $\mathrm{EMS}_{\mathrm{A}}$.)

Traffic jam is an emergent result of interaction of many transport elements and factors (cars, traffic lights, structure of transport symbols, weather, hours in the day time, etc.), $[50,51]$. According to the possible variables in the external description, we consider traffic jam on the highway as a three-dimensional case with the following external variables:

$x_{1}$ : an average number of cars within $1 \mathrm{~km}$

$x_{2}$ : visibility on the road in $[m]$ (in computation the quantity $\left(1 / x_{2}\right)$ is used)

$x_{3}$ : number of bottlenecks (e.g., places of some repairs where it is allowed to use only one road strip) in $10 \mathrm{~km}$ of the road

In the example, we consider calibration constant $\Lambda=1$ and thus $\Delta H_{P}\left(B+1=\Delta H_{D}(B+1)\right.$.

We considered the following nominal and emergent quantities:

$x_{1 \mathrm{nom}}=28$ and $x_{1 \mathrm{em}}=120$. The quotient of importance $\omega_{1}=0.3$

$x_{2 \text { nom }}=100$ and $x_{2 \mathrm{em}}=40$. The quotient of importance $\omega_{2}=0.3$..

$x_{3 \mathrm{nom}}=2$ and $x_{3 \mathrm{em}}=4$. The quotient of importance $\omega_{2}=0.4$.

Applying expressions (10) and (11) (for $\Lambda=1$ ), we compute

$$
\begin{aligned}
\Delta H_{P}(\mathbf{B}+1) & =\left(\left(\omega_{1}\left(\frac{x_{1 \mathrm{em}}}{x_{1 \mathrm{nom}}}\right)\right)^{2}+\left(\omega_{2}\left(\frac{x_{2 \mathrm{em}}}{x_{2 \mathrm{nom}}}\right)\right)^{2}+\left(\omega_{3}\left(\frac{x_{3 \mathrm{em}}}{x_{3 \mathrm{nom}}}\right)\right)^{2}\right)^{1 / 2} \\
& =0.09 * 18.36+0.09 * 6.25+0.16 * 4=1.7 .
\end{aligned}
$$
A.

Now, we use the method for tuning $(u / c)$ from Appendix

For the first estimation of $(u / c)_{0}$, we set up $(u / c)_{0}=0.3$ (from Table 1).

Using expression (9), we compute the first approximation of cardinality of the basis \#

$$
H_{\mathrm{COM}}(\mathbf{B})=\xi\left(\frac{\Delta H_{P}(\mathbf{B}+1)}{(u / c)_{0}}\right)=\xi\left(\frac{1.7}{0.3}\right)=6=\# \mathbf{B} .
$$

Tuning $(u / c)$

For $\# \mathbf{B}=6$, we find in tables [37] Ramsey numbers $R(6$, $3)=18, R(6,4)=[36,42], R(6,5)=[58,87]$, and $R(6,6)=$ [102, 166]. 
For \#B = 7, we find in tables [37] Ramsey numbers $R(7$, $3)=23, R(7,4)=[49,61], R(7,5)=[80,143]$, and $R(7,6)=$ [111, 298].

We are looking for a minimal difference: $\Delta_{0} f(\mathrm{RN})=\min$ $\{(23-18),(49-35),(80-58),(143-87),(111-102)\}-$ smaller differences do not exist\}:

$$
\Delta_{0} f(\mathrm{RN})=5 \text {. }
$$

For the further estimation of $(u / c)_{1}$, we set up $(u / c)_{1}=$ 0.25 (from Table 1):

$$
H_{\mathrm{COM}}(\mathbf{B})=\xi\left(\frac{\Delta H_{P}(\mathbf{B}+1)}{(u / c)_{1}}\right)=\xi\left(\frac{1.7}{0.25}\right)=7=\# \mathbf{B} .
$$

For $\# \mathbf{B}=7$, we find in tables [37] Ramsey numbers $R(7,3)=23, R(7,4)=[49,61], R(7,5)=[80,143]$, and $R(7$, 6) $=[111,298]$

For \# $(\mathbf{B}+1)=8$, we find in tables [37] Ramsey numbers $R(8,3)=28, R(8,4)=[56,84], R(8,5)=[95,216]$, and $R(8$, 6) $=[127,495]$.

We are looking for a minimal difference: $\left.\Delta_{1} f(\mathrm{RN})\right)=\min$ $\{(28-23),(56-49),(95-80),(143-87),(127-111)\}-$ smaller differences do not exist $\}$ :

$$
\Delta_{1} f(\mathrm{RN})=5 .
$$

For the further estimation of $(u / c)_{2}$, we set up $(u / c)_{2}=$ 0.35 (from Table 1).

$$
H_{\mathrm{COM}}(\mathbf{B})=\xi\left(\frac{\Delta H_{P}(\mathbf{B}+1)}{(u / c)_{2}}\right)=\xi\left(\frac{1.7}{0.35}\right)=5=\# \mathbf{B} .
$$

For $\# \mathbf{B}=5$, we find in tables [37] Ramsey numbers $R(5$, $3)=14, R(5,4)=25, R(5,5)=[43,49]$, and $R(5,6)=[58,87]$.

For $\# \mathbf{B}=6$, we find in tables [37] Ramsey numbers $R(6$, $3)=18, R(6,4)=[35,41], R(6,5)=[58,87]$, and $R(6,6)=$ [102, 166].

We are looking for a minimal difference: $\left.\Delta_{2} f(\mathrm{RN})\right)=\min$ $\{(18-14),(35-25),(95-80),(58-49),(102-87)\}-$ smaller differences do not exist\}:

$$
\Delta_{2} f(\mathrm{RN})=4 \text {. }
$$

For the further estimation of $(u / c)_{3}$, we set up $(u / c)_{3}=$ 0.4 (from Table 1).

$$
\left.H_{\mathrm{COM}}(\mathbf{B})=\xi\left(\Delta H_{P} \frac{(\mathbf{B}+1)}{(u / c)_{3}}\right)=\xi\left(\frac{1.7}{0.4}\right)\right)=5=\# \mathbf{B} .
$$

According to (29) and (30), we obtain the same result as for $(u / c)_{3}=0.35$, i.e., $\Delta_{3} f(\mathrm{RN})=4$.

For the further estimation of $(u / c)_{4}$, we set up $(u / c)_{4}=0.45$. $4=\# \mathbf{B}$

$\left.H_{\mathrm{COM}}(\mathbf{B})=\xi\left(\Delta H_{P}(\mathbf{B}+1) /(u / c)_{4}\right)=\xi(1.7) / 0.45\right)=$

$R(4,3)=9, R(4,4)=18, R(4,5)=25, R(4,6)=[35,41]$.

$R(5,3)=14 R(5,4)=25, R(5,5)=[13,49], R(5,6)=[58$, 87].

We are looking for a minimal difference:

$$
\Delta_{4} f(\mathrm{RN})=\min \{(14-9),(25-18),(49-25),(58-35)\}
$$$$
\text { - smaller differences do not exist\} }
$$

TABle 2: Values of components for phases Pbnm and Ibmm.

\begin{tabular}{lcc}
\hline Component $\left(C_{x}\right)$ & Pbnm phase $\left(C_{\text {Pbnm }}\right)$ & Ibmm phase $\left(C_{\text {Ibmm }}\right)$ \\
\hline$\varepsilon_{1}^{x x}(0)$ & 3.234 & 3.273 \\
$\varepsilon_{1}^{y y}(0)$ & 3.137 & 3.173 \\
$\varepsilon_{1}^{z z}(0)$ & 3.116 & 3.079 \\
$\Delta \varepsilon$ & -0.033 & -0.046 \\
$\omega_{p}^{x x}$ & 8.530 & 8.966 \\
$\omega_{p}^{y y}$ & 8.095 & 8.068 \\
$\omega_{p}^{z z}$ & 8.449 & 7.768 \\
$n^{x x}(0)$ & 1.798 & 1.809 \\
$n^{y y}(0)$ & 1.771 & 1.781 \\
$n^{z z}(0)$ & 1.765 & 1.754 \\
$\Delta n(0)$ & -0.030 & -0.041 \\
\hline
\end{tabular}

$\varepsilon_{1}^{x x}(0), \varepsilon_{1}^{y y}(0)$, and $\varepsilon_{1}^{z z}(0)$ are real parts of tensor components of dielectric function in frequency $0 \omega_{p}^{x x}, \omega_{p}^{y y}$, and $\omega_{p}^{z z}$ are real parts of tensor components of plasmon oscillations; $\delta_{\varepsilon}$ is uniaxial anisotropy; $n^{x x}(0), n^{y y}(0)$, and $n^{z z}(0)$ are tensor components of refractive index in frequency $0 ; \Delta n(0)$ represents birefringence.

$$
\begin{aligned}
& \Delta_{4} f(\mathrm{RN})=\min \{5,7,24,23\}=5 \\
& \Delta_{4} f(\mathrm{RN})=5
\end{aligned}
$$

The sequence of values $\left.\Delta_{0} f(\mathrm{RN})\right), \ldots, \Delta_{4} f(\mathrm{RN})$ leads to $\left.\min \left(\Delta_{0} f(\mathrm{RN})\right), \ldots, \Delta_{4} f(\mathrm{RN})\right)=\min (5,5,4,4,5)=4$. And, according to (9), we take the number $(u / c)$ for which we found $\left.\left.\min \left\{\Delta_{0} f(\mathrm{RN})\right), \ldots, \Delta_{3} f(\mathrm{RN}), \Delta_{4} f(\mathrm{RN})\right)\right\}$ and thus $(u / c)=0.35$ OR 0.4 .

We take $(u / c)=0.4$

$$
H_{\mathrm{COM}}(\mathbf{B})=\xi\left(\frac{\Delta H_{P}(\mathbf{B}+1)}{(u / c)_{3}}\right)=\xi\left(\frac{1.7}{0.4}\right)=5=\text { \#. }
$$

For \# $\mathbf{B}=5$, we find in tables [37] Ramsey numbers $R(5$, $3)=14, R(5,4)=25, R(5,5)=[43,49]$, and $R(5,6)=[58,87]$.

For $\# \mathbf{B}=6$, we find in tables [37] Ramsey numbers $R(6$, $3)=18, R(6,4)=[35,41], R(6,5)=[58,87]$, and $R(6,6)=$ $[102,166]$.

$$
\begin{aligned}
\left.\Delta_{\text {opt }} f(\mathrm{RN})\right) \in\{(18-14),(35-25), \\
\\
(95-80),(58-49),(102-87)\} .
\end{aligned}
$$

$\left.\left.\Delta_{\text {opt }} f(\mathrm{RN})\right) \in\{4,10,15,9,15)\right\}$ is the same as we write $\left.\Delta_{\text {opt }} f(\mathrm{RN})\right)=4$ OR 10 OR 15 OR 9.

Figure 3 shows a matroid with a cardinality $X=14$ and with one of the bases of five elements.

The number of edges of the graph in Figure 3 is 91 (some of them are not visible) and 10 of them connect the elements of some base (base marked with red edges).

Furthermore, the extension of the base of the matroid by one element (blue edges) from added four elements is indicated $\left.\Delta_{\text {opt }} f(\mathrm{RN})\right)=4$. In the figure, the connections of these elements with all elements of the matroid are omitted (due to the increased and confusing line density).

7.2.1. Normalization of $\Delta_{o p t} f(R N)$. The additional information about the number of active elements in the actual state of the complex system is $V(\mathbf{B})=800$ (in the compartment) transport elements (cars, traffic lights, structure of transport symbols, etc.). 


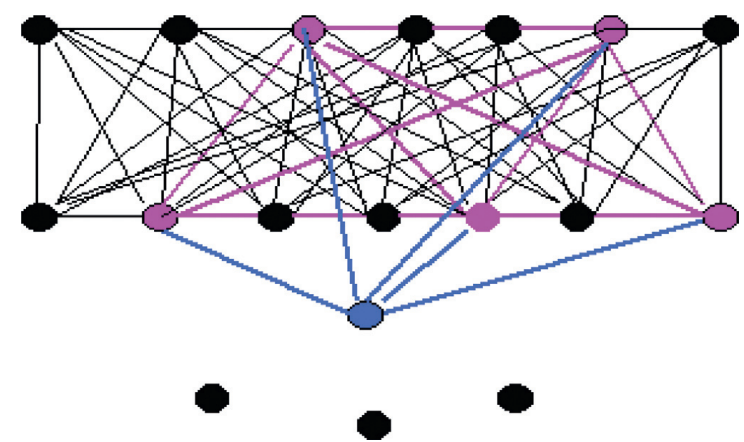

Figure 3: Matroid $M=\langle X, \mathbf{M B}\rangle, \# X=14$, with a marked base $\mathbf{B}, \# \mathbf{B}=5$ and with extension of this base by one element.

$$
\begin{aligned}
\text { For } \mathrm{RN}(\mathbf{B}) & =R(5,3)=14, \\
\lambda & =\frac{V(\mathbf{B})}{\mathrm{RN}(\mathbf{B})} \text { we calculate } \lambda=57.1 .
\end{aligned}
$$

According to (15) and (18) and for $\Delta_{\text {opt }} f(\mathrm{RN}) \in\{4,10,15$, 9)\}, we have

$$
\Delta V(\mathbf{B}+1) \in\{228,571,513,857\} .
$$

The answer to the question about the traffic jam conditions is: The critical contribution of transport elements that could "cause" congestion on the highway is 228,513 , 571 , and 857 (considering the actual state of 800 traffic elements). It means that the complete number of transport elements in the considered compartment of the complex system is $1028,1313,1371$, or 1657 . These numbers only approximate the EMS and "cover" both sudden changes in the structure of the transport system and also something else (invisible).

Note 10. It is hard to provide the reproducibility for emergent situation. It means, e.g., it is not sure if the traffic jam will appear tomorrow among 165 and 298 transport elements as today.

7.3. Phase Transition as an Emergent Phenomenon. The phenomenon of phase transition is usually investigated in chemistry and in crystallography. The selected compound changes its crystallographic structure as a result of changing some external (internal) variables. The causal connection of these variables with phase transition and the proportionality of their influence is usually not completely known and the change of the compound structure is "modelled" as an influence of some representative variable (e.g., temperature-as in our case below). In this section, we investigate the phase transition of $\mathrm{BaThO}_{3}$ between Pbnm crystalographic structure and Ibmm crystallographic structure as an emergent phenomenon.

We use experimental and computed data acquired from [19]. The influence of phase transition on the electronic structure and the optical properties of $\mathrm{BaThO}_{3}$ was investigated by means of Density Functional Theory [19, 52] and verified by program wien2k [53]. At room temperature,
$\mathrm{BaThO}_{3}$ is stable in the $\mathrm{Pbnm}$ phase until $547^{\circ} \mathrm{C}$, whereas it is stable in the Ibmm phase at temperature higher than $700^{\circ} \mathrm{C}$. The transfer from Pbnm phase to Ibmm phase by the influence of temperature is considered an emergent situation. Table 2 presents the quantities for Pbnm phase and Ibmm phase computed by program wien $2 \mathrm{k}$ [53]:

As a power of emergent phenomenon, cumulative result of changes of the optical properties for Pbnm and Ibmm phases are used.

For computation of $\Delta H_{D}(\mathbf{B}+1)$, we use dimensionless expression $\left(C_{\mathrm{Ibmm}} / C_{\mathrm{Pbnm}}\right)$ for all quantities from Table 2; quotients " $\omega_{i}$ " of importance (equation (10)) are equal to 1 and for calibration constant $\Lambda=1$ (equation (11)) and we have $\Delta H_{P}(\mathbf{B}+1)=\Delta H_{D}(\mathbf{B}+1)$.

$$
\begin{aligned}
\Delta H_{D}(\mathbf{B}+1)= & \left(\left(\frac{3.273}{3.234}\right)^{2}+\left(\frac{3.173}{3.137}\right)^{2}+\left(\frac{3.079}{3.116}\right)^{2}+\left(\frac{-0.046}{-0.033}\right)^{2}\right. \\
& +\left(\frac{8.966}{8.530}\right)^{2}+\left(\frac{8.068}{8.095}\right)^{2}+ \\
& \left.+\left(\frac{7.768}{8.449}\right)^{2}+\left(\frac{1.809}{1.798}\right)^{2}+\left(\frac{1.781}{1.77}\right)^{2}+\left(\frac{1.754}{1.765}\right)^{2}\right)^{1 / 2} \\
& +1.1+0.99+0.845+1.01+1.012+0.987)^{1 / 2} \\
\#= & \xi\left(\frac{\Delta H_{P}(\mathbf{B}+1)}{(u / c)}\right)=8 \text { for }\left(\frac{u}{c}\right) \\
= & 0.42 \text { (Table } 1 \text { and the tuning according toAppendix A.) }
\end{aligned}
$$

$\Delta f(\mathrm{RN})=\mathrm{RN}(x, 9)-\mathrm{RN}(y, 8) . \mathrm{RN}(y, 8):(28,56,89,95$, 216, 495). $\mathrm{RN}(x, 9):(36,115,121,316,380)$.

$\Delta f(\mathrm{RN})=\min \quad\{(36-28), \quad(115-28), \quad(115-56), \ldots$, $(115-95), \ldots,(316-95)\}=\min \{8,87,59, \ldots, 20,221\}=8$.

$$
\begin{aligned}
\lambda & =\frac{547}{28}=19.5, \\
\Delta T & =\lambda \cdot \Delta f(\mathrm{RN})=19.5 * 8=156^{\circ} \mathrm{C} .
\end{aligned}
$$

The quantity of temperature that is needed for the considered phase transfer is

$$
T_{\mathrm{PT}}=547+156=703^{\circ} \mathrm{C} \text {. }
$$

And, it corresponds to results of experimental verification, the phase transition, and its consequences [19].

We may compare this result with calculation in [24], where

$$
\begin{aligned}
\# \mathbf{B} & =\xi\left(\Delta H_{P} \frac{(\mathbf{B}+1)}{(u / c)}\right)=9 \text { for }\left(\frac{u}{c}\right) \\
& =0.37 \text { (Table } 1 \text { and the tuning according to Appendix A.), }
\end{aligned}
$$

$\Delta T=161.84^{\circ} \mathrm{C}$

The quantity of temperature that is needed for the considered phase transfer is 


$$
T_{\mathrm{PT}}=547+161.84=708.84^{\circ} \mathrm{C} \text {. }
$$

Both cases lead to a temperature higher than $700^{\circ} \mathrm{C}$, and it corresponds to results of experimental verification of the phase transition, [19].

\section{Discussion}

In this section, only some substantial issues of our developed method and its application are introduced:

The structural invariants are defined by expressions (1) and (2).

Calculating \#B and $\mathrm{RN}(\# \mathbf{B}, Y)$ in expression (12), we consider a perfect subgraph with \#B nodes in a perfect graph $G_{p}$ with $\mathrm{RN}(\# \mathbf{B}, Y)$ nodes. This case leads to the calculation of $\Delta f(\mathrm{RN})$ and to $\Delta V(\mathbf{B}+1)$ in normalization procedure. However, this is only one variant where the matroid is associated with a perfect graph. There are other graph representations of the matroid with \#B node basis (e.g., in [35]).

Additive Representation of Drivers. The PAES that corresponds to values of symptoms concentrated in the power of the emergent phenomenon $\left(\Delta H_{P}(\mathbf{B}+1)\right)$ is associated with the result of the normalization procedure $\Delta V(\mathbf{B}+1)$. In the "volume" $\Delta V(\mathbf{B}+1)$ of real elements, it is necessary to add to a current volume $\mathrm{V}(\mathrm{B})$ in order to model a possible appearance of emergent situation (PAES). We are speaking about additive representation of drivers. This is the only one way to use $\# \mathbf{B}$ and to construct matroid $M\left(X, \mathbf{B}_{1}, \ldots, \mathbf{B}_{n}\right)$.

Verifiability and Reproducibility. The results obtained using the method submitted are not verifiable in the classical sense. E.g., if we calculated today that with connecting 98 new water carriers to 300 existing ones, will be associated an emergent flood situation, it may not happen tomorrow or next month.

\section{Conclusion}

The whole article is based on the thesis that emergent phenomena in complex systems are associated with sharp changes in the structure of the systems, with coincidence of events in the systems (in the NAT plane) or with something else (S.E.) - what we do not actually see. However, we are able to record and to process these changes in some symbolical system (in SYMB). For the analysis and the detection of emergent situations, we used the so-called structural invariants in SYMB by which we are able to indicate the eventual occurrence of the emergence as their violation. In the article, a list of structural invariants that we have discovered in the analysis and in the detection of emergent situations is introduced. This list is not limited by cases from physics (where we have started with R. Laughlin [13]). It focuses in particular on emergences on macrostructures, such networks (hydrological and transport networks $[31,32])$, ecosystems or bioengineering systems monitoring [6], and processes with characteristic cycles (cycles in machines, biorhythms, circadian cycles, ECG signals $[43,44])$.
In this article, we focused on the development of a method for the analysis of emergent situations with the help of a structural invariant $(\mathbf{M}, \mathbf{B M})$ and its application on macrostructures (Sections 7.1 and 7.2) and on microstructures (Section 7.3).

\section{Appendix}

The method of soft tuning of $(u / c)$ is as follows: considering the complexity of the compartment of the complex system given by the number of a matroid- $-H_{\mathrm{COM}}$ $(\mathbf{B})=\# \mathbf{B}$-the procedure for calculating and determining the coefficient $(u / c)$ is based on the following limitations and conditions:

$$
\begin{aligned}
& \text { (a) }\left(\frac{u}{c}\right) \in\langle 0,1\rangle, \\
& \text { (b) } \Delta H_{P}(\mathbf{B}+1) \geq 0.1 \text {, } \\
& \text { (c) } \# \mathbf{B} \in Z^{+} \text {. }
\end{aligned}
$$

Procedure for computation of $(u / c)$ :

(S1) First, we compute $\Delta H_{P}(\mathbf{B}+1)$ (resp., $\Delta H_{D}(\mathbf{B}+1)$ ).

(S2) Fulfilling condition $(u / c) \in\langle 0,1\rangle$ (starting with an initial quantity of $(u / c)$-for orientation, it is possible to use estimations from Table 1) is chosen the quantity $\# \mathbf{B}$.

(S3) In the list of Ramsey numbers [37], we find numbers $R(3, \# \mathbf{B}), \quad R(4, \# \mathbf{B}), \ldots, R(r 1, \quad \# \mathbf{B})$. (Numbers $R(\ldots, \# \mathbf{B})$ are sometimes given as subsets on the interval $[R 1, R 2] \in Z^{+}$).

(S4) We find the nearest numbers $R(3, \# \mathbf{B}+1), R(4$, $\# \mathbf{B}+1), \ldots, R(r 2, \# \mathbf{B}+1)$.

(S5) We compute combinations of Ramsey numbers for the selection of a minimal difference:

$\Delta f(\mathrm{RN})=\mathrm{RN}(x, \# \mathbf{B}+1)-\mathrm{RN}(y, \# \mathbf{B})$.

So we find numbers $\mathrm{RN}(y, \# \mathbf{B})$ : $\left(p_{1}, p_{2}, p_{3}\right.$, $\left.p_{4}, p_{5}, \ldots\right)$, and $\mathrm{RN}(x, \# \mathbf{B}+1):\left(q_{1}, q_{2}, q_{3}, q_{4}, q_{5}\right.$, etc.), and we express the sequence $\left(\left(q_{1}-p_{1}\right)\right.$, $\left.\left(q_{1}-p_{2}\right),\left(q_{2}-p_{2}\right),\left(q_{2}-p_{3}\right),\left(q_{3}-p_{3}\right), \ldots\right)$

(S6) From this sequence is computed $\Delta_{1} f(\mathrm{RN})=$ $\min \left\{\left(q_{1}-p_{1}\right),\left(q_{1}-p_{2}\right),\left(q_{2}-p_{2}\right),\left(q_{2}-p_{3}\right), \quad\left(q_{3}\right.\right.$ $\left.\left.-p_{3}\right), \ldots\right\}$.

(S7) We turn back to step S2 and we select another $(u / c)$ (fulfilling condition a).

(S8) We continue till the step S6 and compute $\left.\Delta_{2} f(\mathrm{RN})\right)=\min \{\ldots\}$.

(S9) This procedure from S7 to S8 is repeated as many times as possible until the variability of the sequence $\left(\Delta_{1} f(\mathrm{RN}), \ldots, \Delta_{k} f(\mathrm{RN})\right)$ is seen.

(S10) The best value of the quotient $(u / c)$ is a number for which we found

$$
\min \left\{\left(\Delta_{1} f(\mathrm{RN})\right), \ldots,\left(\Delta_{k} f(\mathrm{RN})\right)\right\} \text {. }
$$


(S11) For this value of $(u / c)$ we solve equations (9) and (12) (from Section 6.2) and we continue in the method.

\section{Data Availability}

This article contains results of an applied theory. All substantial sources are introduced in references of the proposed paper.

\section{Conflicts of Interest}

The authors declare that they have no conflicts of interest.

\section{Acknowledgments}

This research was funded by Research Grant RVO 12000 institutional support for the development of a research organization, and the authors are grateful for the support.

\section{References}

[1] M. Kotyrba, E. Volna, and P. Bujok, "Unconventional modelling of complex system via cellular automata and differential evolution," Swarm and Evolutionary Computation, vol. 25, pp. 52-62, 2015.

[2] I. Navarro and F. Matía, "An introduction to swarm robotics," ISRN Robotics, vol. 2013, pp. 1-10, 2013.

[3] M. D. Petty, "Modeling and validation challenges for complex systems," MઐS Journal, vol. 9, no. 1, pp. 25-35, 2014.

[4] A. F. Siegenfeld and Y. Bar-Yam, "An introduction to complex systems science and its applications," Complexity, vol. 2020, Article ID 610852, , 2020.

[5] J. Bila, R. J. Rodríguez, and M. Novak, "Modeling of complex systems by means of partial algebras," Mendel, vol. 25, no. 1, pp. 103-110, 2019.

[6] J. Bila, J. Jura, J. Pokorny, and I. Bukovsky, "Qualitative modeling and monitoring of selected ecosystem functions," Ecological Modelling, vol. 222, no. 19, pp. 3640-3650, 2011.

[7] V. Darley, "Emergent phenomena and complexity," in Proceedings of the Fourth International Workshop on the Synthesis and Simulation of Living Systems, R. A. Brooks and P. Maes, Eds., The MIT Press, Cambridge, MA, USA, pp. 411-416, 1996.

[8] W. Kinsner, "Complexity and its measures in cognitive and other complex systems,", in ICCI08, pp. 2-75, Stanford, CA, 2008.

[9] I. Bukovský, W. Kinsner, and J. Bíla, "Multiscale Analysis approach for novelty detection in adaptation plot," in Sensor Signal Processing for Defence 2012, pp. 1-6, IET Publishing Group, Stevenage, UK, 2012.

[10] J. Bila, "The detection of emergent situations by structural invariants,", in Proceedings of the 17th International Conference on Soft Computing, MENDEL, R. Matousek, Ed., vol. 17, pp. 534-539, Brno University of Technology, VUT Press, Brno, Czech Republic, 2011.

[11] J. Johnson and J. J. Padilla, "Ontology of emergence," in In Engineering Emergence: Modeling and Simulation Approach, L. Rainey and Mo. Jamshidi, Eds., pp. 185-198, CRS Press, Taylor and Francis, Boca Raton, FL, USA, 2019.

[12] J. B. Rainey and Mo. Jamshidi, Engineering Emergence. A Modeling and Simulation Approach, CRC Press, Taylor \& Francis, Boca Raton, FL, USA, 2019.
[13] R. B. Laughlin, A Different Universe (Reinventing Physics from the Bottom Down, Basic Books, New York, NY, USA, 2006.

[14] R. Cottam, W. Ranson, and R. Vounckx, "Emergence: half a quantum jump," Acta Polytechnica Scandinavica. Emergence, Complexity, Hierarchy. Order, Espoo: Finish Academy of Technology, pp. 12-19, 1998.

[15] J. Bila, "Emergent phenomena in complex systems, recent advances in soft computing," Advances in Intelligent Systems and Computing, Springer, vol. 837, , pp. 262-270, 2019.

[16] F. Jensen, Introduction to Computational Chemistry, Wiley, New York, NY, USA, 1999.

[17] A. H. Reshak, S. A. Khan, H. Kamarudin, and J. Bila, "NaAuS chicken-wire-like semiconductor: electronic structure and optical properties," Journal of Alloys and Compounds, vol. 582, pp. 6-11, 2014.

[18] A. H. Reshak, Z. A. Alahmed, J. Bila et al., "Exploration of the electronic structure of monoclinic $\alpha$-Eu2(MoO4)3: DFTbased study and X-ray photoelectron spectroscopy," The Journal of Physical Chemistry C, vol. 120, no. 19, pp. 1055910568, 2016.

[19] A. H. Reshak, Z. A. Alahmed, and J. Bila, "Phase transition in $\mathrm{BaThO} 3$ from Pbnm to Ibmm turn the fundamental energy band gap from indirect to direct," Journal of Alloys and Compounds, vol. 771, pp. 607-613, 2019.

[20] P. Horava and E. Witten, Nuclear Physics, vol. 460, pp. 506-524, 1996.

[21] P. Draper, P. Meade, M. Reece, and D. Shih, "Implication of a $125 \mathrm{GeV}$ Higgs for the MSSM and low-scale SUSY breaking," Physical Review D.vol. 85, no. 9, Article ID 095007, 2011.

[22] S. Weinberg, "The quantum theory of fields," Supersymmetry, Vol. 3, Cambridge University Press, Cambridge, UK, 1999.

[23] C. G. Jung and W. Pauli, Naturerklärung und Psyche, JungInstitut, Zürich, Switzerland, 1952.

[24] J. Bila, A. H. Reshak, and J. Chysky, "Phase Transition as an Emergernt Phenomenon analysed by violation of structural invariant (M, BM)," MENDEL-Soft Computing Journal, vol. 26, no. 2, pp. 45-50, 2020.

[25] H. Haken and M. Wagner, Cooperative Phenomena, Springer, Heidelberg, Germany, 1973.

[26] R. G. B. Reid and "B. Emergences, Evolution by Natural Experiment, Massachusetts Institute of Technology, Massachusetts, FL, USA, 2007.

[27] T. Holland, "Foundations for the modeling and simulation of emergent behavior systems," in Engineering Emergence: Modeling and Simulation Approach, I. R. M. Jamshidi, Ed., CRS Press/Taylor and Francis, Boca Raton, FL, USA, pp. 217-258, 2018.

[28] S. A. Kauffman, Investigations, Oxford University Press, Oxford, UK, 2000.

[29] V. V. Kryssanov, H. Tamaki, and S. Kitamura, "Understanding design fundamentals: how synthesis and analysis drive creativity, resulting in emergence," Artificial Intelligence in Engineering, vol. 15, no. 4, pp. 329-342, 2001.

[30] J. Bila, L. Koran, and R. Mankova, "Detection of ill separable faults,", in Proceedings of 4th IFAC Symposium on Fault Detection, Supervision and Safety for Technological Proceses - SAFEPROCES 2000, pp. 461-465, Budapest, Hungary, 2000.

[31] J. Bila, "Emergent phenomena in complex systems and their detection," International Journal of Enhanced Research in Science Technology and Engineering, vol. 6, no. 12, pp. 40-53, 2017.

[32] J. Bila, "Emergent phenomena in natural complex systems," in Emergence, Complexity and Computation, A. Sanayei, I. Zelinka, and O. R. Rőssler, Eds., in Proceedings of 
Interdisciplinary Symposium on Complex Systems, pp. 89-100, Springer, Heidelberg, Germany, 2014.

[33] B. Hannisdal and L. H. Liow, "Causality from palaeontological time series," Palaeontology, vol. 61, no. 4, pp. 495-509, 2018.

[34] G. Sugihara, R. May, H. Ye et al., "Detecting causality in complex ecosystems," Science, vol. 338, no. 6106, pp. 496-500, 2012.

[35] J. G. Oxley, Matroid Theory, Oxford Science Publications, Oxford, UK, 2001.

[36] F. P. Ramsey, "On a problem of formal logic," Proceedings of the London Mathematical Society, vol. s2-30, no. 1, pp. 264286, 1930.

[37] W. Weinstein, "Ramsey numbers," A Wolfram Web Resource, https://mathword.wolfram.com/RamseyNumber.html, May 2004.

[38] L. Fern, G. Gordon, J. Leasure, and S. Pronchik, "Matroid automorphisms and symmetry groups, combinatorics, probability and computing," vol. 9, pp. 105-123, 2001.

[39] D. Bohm, Wholeness and the Implicate Order, Routledge, London, UK, 1980.

[40] R. A. Rodríguez, R. Riera, A. M. Herrera et al., "Degrees of freedom: definitions and their minimum and most meaningful combination for the modelling of ecosystem dynamics with the help of physical principles," Ecological Modelling, vol. 392, pp. 226-235, 2019.

[41] P. J. F. M. Verweij, M. J. R. Knapen, W. P. de Winter et al., “An IT perspective on integrated environmental modelling: the SIAT case," Ecological Modelling, vol. 221, no. 18, pp. 2167-2176, 2010.

[42] J. Bila and M- Tlapak, "Knowledge discoveries and emergent synthesis in conceptual ReDesign process,", in Proceedings of the International Conference on Intelligence for Modelling, Control and Automation, pp. 252-260, IEEE, Sydney, Australia, December 2006.

[43] J. Bila and M. Mironovova, "Interpretation of new ECG signal shapes for diagnostics of cardio-vascular system," in Proceedings of the 10th International Conference on P2P, Parallel, Grid, Cloud and Internet Computing, pp. 386-391, IEEE, Warsaw, Poland, 2015.

[44] J. Bila and M. Novák, "The detection of coming some diseases as emergent situations in complex systems," in Proceedings of the Mendel 2016 22nd International Conference on Soft Computing, pp. 281-288, Brno: Brno University of Technology, Faculty of Mechanical Engineering, Brno, Czech Republic, June 2016.

[45] F. Matus, "Abstract functional dependency structures," Theoretical Computer Science, vol. 81, pp. 117-126, 1991.

[46] T. L. Saaty, "Exploring the interface between hierarchies, multiple objectives and fuzzy sets," Fuzzy Sets and Systems, vol. 1, no. 1, pp. 57-68, 1978.

[47] R. Beranova, J. Kysely, and M. Hanel, "“Characteristics of subdaily precipitation extremes in observed data and regional climate model simulations," Theoretical and Applied Climatology, vol. 132, no. 1-2, pp. 515-527, 2018.

[48] V. Svoboda, M. Hanel, P. Máca, and J. Kyselý, "Characteristics of rainfall events in regional climate model simulations for the Czech Republic," Hydrology and Earth System Sciences, vol. 21, no. 2, pp. 963-980, 2017.

[49] Q. Yao, J. Xie, L. Guo, X. Zhang, and R. Liu, "Analysis and evaluation of flash flood disasters: a case of Lingbao county of Henan province in China," Procedia Engineering, vol. 154, pp. 835-843, 2016.

[50] K. Nagel and S. Rasmussen, "Traffic at the edge of chaos," in Proceedings of the Fourth International Workshop on the
Synthesis and Simulation of Living Systems, R. A. Brooks and P. Maes, Eds., The MIT Press, Cambridge, MA, USA, pp. 236-245, 1996.

[51] L. Zhang, G. Zeng, D. Li, H.-J. Huang, H. E. Stanley, and S. Havlin, "Scale-free resilience of real traffic jams," Proceedings of the National Academy of Sciences, vol. 116, no. 18, pp. 8673-8678, 2019.

[52] P. Hohenberg and W. Kohn, "Inhomogeneous electron gas," Physical Review, vol. 136, no. 3B, Article ID B864, 1964.

[53] P. Blaha, K. Schwarz, G. Madses, D. Kvasnicka, and J. Luitz, "wien $2 \mathrm{k}$, an augmented plane wave -local orbitals program for calculating crystal properties," 2001. 